\title{
MAOB rs3027452 Modifies Mood Improvement After Tryptophan Supplementation
}

\author{
Irene Gonzalez (D) \\ Rocio Polvillo ${ }^{1,2}$ \\ Maximiliano Ruiz-Galdon' \\ Armando Reyes-Engel' \\ Jose Luis Royo' \\ 'Department of Surgery, Biochemistry \\ and Immunology School of Medicine, \\ University of Malaga, Malaga, Spain; \\ ${ }^{2}$ Centro Andaluz de Biología del \\ Desarrollo, Seville, 41013, Spain
}

\begin{abstract}
Purpose: Tryptophan is the only precursor of serotonin, the hormone which helps regulate key human functions such as appetite, memory, mood, and sexual behavior. Connections have been identified between serotonin system dysfunction and the molecular etiology and treatment of mood disorders in a wide range of studies. Proposals have been put forward to co-administer tryptophan supplementation together with serotonin reuptake inhibitors in major depression patients, and also to exploit the sub-therapeutic depressive status in healthy populations. The reported responses, however, have been very dissimilar and this uneven effect may largely be explained by interindividual genetic differences.

Materials and Methods: We studied mood change in 138 healthy subjects using both Goldberg's General Health Questionnaire and the Profile of Mood States Questionnaire to determine the effects of a daily supplementation of $1 \mathrm{~g}$ of tryptophan or placebo. Buccal DNA samples were provided and TPHI (rs1800532), MAOA (rs3788862 and rs979605), MAOB (rs3027452), and COMT (rs6269 and rs4680) variants were genotyped.
\end{abstract}

Results: $M A O B$ rs3027452 was equally associated with tryptophan supplementation efficacy in the depression subscales of both questionnaires $(\Delta \mathrm{T}$-Score.D; $\Delta \mathrm{T}$-Score.TMD and $\triangle$ POMS.D p-values $<0.01$ ).

Conclusion: Here we provide evidence that tryptophan supplementation has an uneven effect on mood improvement in the general population.

Keywords: tryptophan, serotonin, 5-HT, mood, pharmacogenetics, monoamine oxidase

\section{Introduction}

Tryptophan (Trp) is an essential amino acid that has to be ingested through protein-rich foods. Plasma Trp levels are the result of both intake and plasma clearance rates. ${ }^{1,2} \operatorname{Trp}$ is the only precursor of serotonin (5-hydroxytryptamine or 5-HT), which is produced both peripherally and centrally. In the central nervous system, 5-HT regulates key functions such as appetite, memory, mood, and sexual activity. ${ }^{3}$ The implication of 5-HT in the pathophysiology and treatment of mood disorders has been studied extensively. Mood disturbance is one of the main symptoms of depression, an affective disorder which is the leading cause of disability worldwide. ${ }^{4}$ The main therapeutic agents used to treat depression are antidepressants like 5-HT reuptake inhibitors, which can be prescribed alone or in combination with serotonin/noradrenaline reuptake inhibitors. ${ }^{5}$ Selective serotonin reuptake inhibitors (SSRIs) act by inhibiting the reuptake of 5-HT into presynaptic cells and thus increasing its extracellular concentration. This increases the amount of 5-HT in the synaptic cleft, binding it to the postsynaptic receptors. SSRIs display different degrees of selectivity for other monoamines such as dopamine and norepinephrine transporters. ${ }^{6}$ The benefits of increased monoamine
Correspondence: Armando Reyes-Engel; Jose Luis Royo

Tel +34 952I3I53I; +34 952136655

Email engel@uma.es; jIroyo@uma.es 
levels were discovered in the 1950s, when monoamine oxidase (MAO) inhibitors and tricyclic antidepressants were found to be efficient in treating major depression patients. This led to the hypothesis that depression was caused by a deficiency in monoamine neurotransmitters. ${ }^{7}$ However, antidepressants are only partially effective in the treatment of moderate to severe depression in adults, suggesting that potential pharmacogenetic effects may condition treatment efficiency. $^{7-11}$

The benefits of Trp for mood were first reported in the late fifties by Lauer et al $1958^{12}$. Some early studies conducted with depressed patients found that a daily supplementation of 3.5-18 g of Trp enhanced the therapeutic benefits of MAO inhibitors and tricyclic antidepressants. ${ }^{13-15}$ However, although effective in mild to moderately depressed patients, Trp supplementation has been shown to have little or no effect in severely depressed patients. ${ }^{16}$ The effects of Trp supplementation on mood in healthy volunteers have also been very varied. Markus et al investigated the effects of different sources of Trp on healthy subjects using the profile of mood states (POMS) questionnaire. ${ }^{17}$ Although the total mood disturbance score was found to improve significantly $60 \mathrm{~min}$ after Trp intake, the maximum score was observed $210 \mathrm{~min}$ after Trp administration. That study, however, lacked longterm evaluation to determine more enduring effects, and the patients in the sample were found to be very heterogeneous. ${ }^{18}$ A significant proportion of this variance might be attributed to pharmacogenetics, and the germline variants involved in Trp and 5-HT metabolisms may have a considerable impact on behavior. We therefore focused this study into the role of the transporters and enzymes involved in the 5-HT metabolism on the way tryptophan supplementation affects mood improvement. To this end, we analyzed the mood states of a series of healthy subjects before and after the administration of Trp/placebo, mapping four genes from the 5-HT metabolism in order to study six germline variants.

\section{Materials and Methods Study Population}

The study population comprised 138 students from the School of Medicine at the University of Malaga. Inclusion criteria included being $>18$ years old, and having no self-reported psychiatric disease. Participants were asked to fill out a questionnaire covering general anthropometric variables and whether or not they were currently receiving any drug treatment. The series was randomly split into two groups: 113 participants who blindly received a 15-day dietary supplementation of 2 capsules with $500 \mathrm{mg}$ of tryptophan (Guinama Laboratories, Malaga, Spain), and twenty-five subjects who received placebo. At the beginning and at the end of the treatment, the participants were asked to complete two selfadministered online tests: the Goldberg General Health Questionnaire (GHQ-28) and the Profile of Mood States (POMS) test. GHQ-28 basically comprises 28 items designed to evaluate four subscales, although we focused specifically on the Depression score. ${ }^{19}$ The POMS questionnaire consists of 48 items, designed to compute six affective states. For each state, a T-Score is calculated depending on the standard deviation and the mean of the variable. We were particularly interested in the Depression (T-Score.D) subscale. Total Mood Disturbance (TMD) represents a global score, since it is calculated by adding negative moods and subtracting positive emotional responses. ${ }^{20}$ This study was performed with the approval of the Ethics Committee of the University of Malaga. All the participants gave their informed consent in writing, in accordance with the principles stated in the Declaration of Helsinki.

\section{DNA Extraction and Genotyping}

DNA was first extracted from each donor by buccal swab. The first Trp/placebo intake was then started using conventional proteinase-K digestion followed by ethanol precipitation. Sample quality and quantity were calculated with Nanodrop ${ }^{\mathrm{TM}}$ technology. The following genes and SNPs were selected: TPHI (rs1800532), MAOA (rs3788862 and rs979605), MAOB (rs3027452), and COMT (rs6269 and rs4680). Genotyping was performed using the TaqMan Open Array Genotyping System from the Applied Biosystems facilities at Genologica SL (Malaga, Spain).

\section{Statistical Analysis}

To determine whether genotypes fitted the HardyWeinberg equilibrium we used the Institute of Human Genetics (Munich) web services (https://ihg.gsf.de/ihg/ snps.html). Statistical analysis was carried out with IBM SPSS Statistics v22. Differences in the different depression subscales were calculated as the final score minus the initial score. The linear regression analysis was adjusted by baseline, weight, sex and age. The level of significance was 0.05 . 


\section{Results}

One hundred and thirty-eight participants were recruited, all of Caucasian origin. The group had an average age of 21.7 years [range: 19-50], and an over-representation of females (62\%). Average BMI was 22.4 [range: 19-39]. Of the initial volunteers, 58 (42\%) completed only the initial evaluation and were not therefore included in the analysis. The Trp supplemented group had a drop-out rate of $40.7 \%$, while the placebo group had a drop-out rate of $48 \%$. This increase might be attributable to a lack of any perceived improvement, but in any case this variance is not statistically significant $\left(\chi^{2} \mathrm{p}\right.$-value $\left.=0.655\right)$. No differences were observed in the baseline levels of either the placebo or Trp groups for any of the variables calculated from the POMS and CGH-28 tests. Neither were any statistical differences found between the placebo and the Trp supplemented group for sex, age, or body-mass index (BMI). When the variants under analysis were examined, the SNPs mapping to autosomes fitted the Hardy-Weinberg equilibrium (Supplementary Table 1).

Increment $(\Delta)$ for each subscale under study was calculated for the volunteers who completed both questionnaires at the beginning and the end of the treatment. Interdependence between the different subscales from the GHQ-28 and POMS questionnaires was also studied. As expected, opposite subscales such as Vigor and Depression in GHQ-28 correlated negatively, while the Anger, Depression, and Tension subscales correlated positively (Supplementary Table 2). Overall, both tests showed a high degree of concordance. We next analyzed the extent to which the observed $\Delta \mathrm{T}$-Scores were conditioned by the basal levels (Supplementary Table 3). We found that, in general, fold change over any subscale largely depended on the starting point. For instance, GHQ-28 Total Mood Disturbance (T-Score.TMD) correlated negatively with the $\Delta \mathrm{T}$ - Score.TMD $(\mathrm{Rho}=-0.464, \quad$ Spearman's $\mathrm{p}$-value
$<0.001$ ), suggesting that the potential benefit of the supplementation was uneven and the lower the starting point, the better the mood recovery. For this reason, basal levels were also included in the regression analysis. When the efficiency of the Trp supplementation was evaluated, we observed that GHQ-28 subscales $\Delta \mathrm{T}$-Score.TMD and $\Delta \mathrm{T}$ Score.D and $\triangle$ POMS.D were higher in the placebo group, suggesting that the Trp supplementation was effective. Although a clear trend was observed, however, the results were not statistically significant (Table 1 ). The high variance found in the results suggested that the treatment might not have been equally effective among the different volunteers. When SNPs were included in the analysis, $M A O B$ rs3027452 appeared to correlate with the efficiency of the Trp supplementation (Table 2), subjects with $M A O B$ rs3027452 AA genotypes displaying lower treatment efficacy (Figure 1). The most common genotype (GG) had $\triangle$ POMS.D and $\Delta \mathrm{T}$-Score.TMD values of -0.45 and -6.83 , respectively, in contrast to the 0.00 and -1.85 shown by the mutant genotype (TT) (Supplementary Table 4).

\section{Discussion}

Trp levels are directly related to 5-HT levels through the synthesis, distribution, and catabolism of both Trp itself and 5-HT. The effect of tryptophan supplementation on mood has been evaluated in numerous studies. The metabolism or synthesis of 5-HT, its transport, and its catabolism are mediated by various polymorphic genes.

It has been observed that $90 \%$ of $5-\mathrm{HT}$ is found in the intestine, $5 \%$ in the brain and the other $5 \%$ in blood, where it is stored in the platelets. ${ }^{21-23}$ This distribution is mediated by multiple factors: firstly, the absorption and the conversion rate of 5-HT in the intestine; secondly, the synthesis of 5-HT in peripheral tissues and blood, stored massively in platelets; and thirdly, the catabolism of tryptophan itself by 2,3-dioxygenase and tryptophan

Table I Effect of Trp Supplementation on the GHQ-28 and POMS Depression Subscales

\begin{tabular}{|c|c|c|c|c|c|}
\hline Test & Subscale & $\begin{array}{c}\text { Placebo } \\
(n=13) \\
\text { (Average } \pm \text { Standard } \\
\text { Deviation) }\end{array}$ & $\begin{array}{c}\text { Trp Supplementation } \\
\qquad(\mathrm{n}=67) \\
\text { (Average } \pm \text { Standard } \\
\text { Deviation) }\end{array}$ & Univariant* & Adjusted $^{+}$ \\
\hline \multirow[t]{2}{*}{ GHQ-28 } & $\Delta \mathrm{T}$-Score.D & $2.49 \pm|4.3|$ & $-3.61 \pm 10.88$ & 0.071 & 0.272 \\
\hline & $\Delta \mathrm{T}$-Score.TMD & $-0.29 \pm 12.07$ & $-5.06 \pm 10.77$ & 0.096 & 0.475 \\
\hline POMS & $\triangle P O M S . D$ & $0.31 \pm 2.72$ & $-0.30 \pm 1.37$ & 0.500 & 0.203 \\
\hline
\end{tabular}

Notes: Effect of Trp supplementation measured with the Goldberg's General Health Questionnaire (GHQ-28) and the Profile of Mood States (POMS) tests. Increments measured in the GHQ-28 Depression (T-Score.D) and Total Mood Disturbance (T-Score.TMD) subscales, and in the POMS Depression subscale (POMS.D). *U-Mann Whitney $\mathrm{p}$-value, ${ }^{+}$Adjusted by baseline, weight, sex and age. 
Table 2 Multivariate Analysis of Trp Supplementation Efficiency Determined Using GHQ-28 and POMS Depression Subscales

\begin{tabular}{|l|c|c|c|c|c|c|c|c|c|}
\hline & \multicolumn{3}{|c|}{$\Delta$ T-Score.D } & \multicolumn{3}{c|}{$\Delta$ T-Score.TMD } & \multicolumn{3}{c|}{$\Delta$ POMS.D } \\
\cline { 2 - 10 } & Beta & t & p-value & Beta & t & p-value & Beta & t & p-value \\
\hline Gender & -0.170 & -1.353 & 0.181 & -0.065 & -0.488 & 0.627 & -0.161 & -1.616 & 0.111 \\
Weight & -0.135 & -1.032 & 0.306 & -0.093 & -0.686 & 0.495 & -0.134 & -1.253 & 0.215 \\
Basal levels & -0.470 & -4.482 & $<0.001$ & -0.384 & -3.515 & $<0.001$ & -0.666 & 7.729 & $<0.001$ \\
MAOB rs3027452 & 0.297 & 2.782 & 0.007 & 0.349 & 3.091 & 0.003 & 0.294 & 3.578 & $<0.001$ \\
\hline
\end{tabular}

Notes: Variables under analysis were the increments $(\Delta)$ measured after treatment using Goldberg's Depression (T-Score.D) and Total Mood Disturbance (T-Score.TMD) subscales, and the POMS Depression subscale (POMS.D). Sample size $=80$.

2,3-dioxygenase, resulting in kynurenine and then niacin formation. These factors, together with the blood-brain barrier's restricting of the passage of solutes, appear to regulate the imbalance in concentrations between the CNS and peripheral tissues, acting as a protective barrier against the negative effects of high concentrations in nerve tissue.

In our study, we were unable to fully determine the direct effect of Trp supplementation on mood in a healthy population, although there was a general tendency towards improvement. Our polymorphism-based analysis of the population revealed a differential response distribution in two functional polymorphisms. Both $M A O A$ and $M A O B$ polymorphisms were seen to compromise Trp-associated mood improvement. According to the eQTL shown for $M A O B$ at the GTEx portal, this enzyme presents greater expression in neural tissues than the $M A O A$ type (Supplementary Figure 1). This contradicts the assumption of a peripheral role for MAOB and a central nervous system role for MAOA. ${ }^{24-26}$ These differences are not invaluable but constitute a unit of magnitude in the CNS, presupposing that MAOB could play a more important role in the clearance and catabolism of neurotransmitters at neurological level than MAOA. MAOB activity at the peripheral level, measured in whole blood, is very low in relation to the other tissues. This corresponds to its high concentration in intestines and platelets, which may serve to maintain high levels of intestinal and platelet 5-HT but which has not yet been fully explained. The MAOB rs3027452 TT genotype represents the lowest transcription rates in the CNS, which would correlate with lower activity and coincide with an increase in 5-HT concentration (Supplementary Figure 2). This genotype has a very negative mood response to tryptophan treatment which could be related to an excessive increase in 5-HT. The molecular basis of the influence of the MAOB polymorphism on mood states observed during Trp supplementation will therefore not be due to the direct effect of the MAOB on the CNS but to the increased amount of Trp available to cross the CNS brain-blood barrier, caused by the lower activity of the peripheral MAOB rs3027452 TT genotype.

A similar effect, already mentioned above, was found with the 5-HTTLPR polymorphism of the 5-HT transporter. This has been associated with a gender-related negative effect dependent on treatment with tryptophan. ${ }^{16}$ Finally, it should be noted that our study has some important limitations. The small sample size and the short-term intervention explain the small magnitude of effect observed in the Trpsupplemented group. Also, some confounders will be recorded in future experiments, such as physical activity, alcohol consumption and pregnancy status.
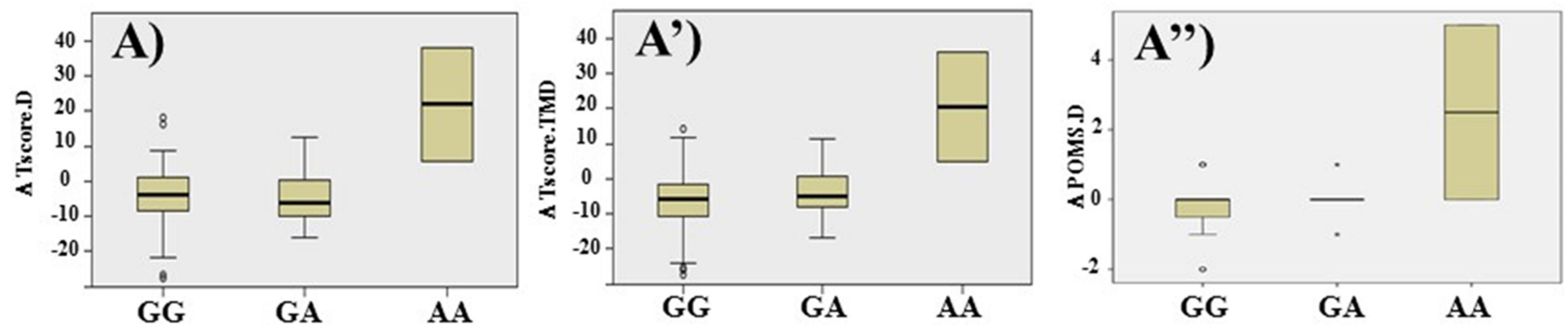

Figure I Box plots reflecting the Trp effect on depression subscales (A) $\Delta$ T-Score.D; (A") $\Delta$ T-Score.TMD and (A") $\Delta . P O M S . D$, according to the MAOB rs3027452 genotype. 


\section{Conclusion}

Our results highlight a pharmacogenetic component of Trp supplementation efficacy, focusing on the contribution of both of the $M A O B$ genetic variants. In order to independently confirm this effect, however, future studies will be carried out over a longer monitoring period and with a larger sample.

\section{Abbreviations}

TRP, tryptophan; 5-HT, 5-hydroxytryptamine; SSRIs, selective serotonin reuptake inhibitors; POMS, profile of mood state; GHQ-28, Goldberg general health questionnaire; TMD, total mood disturbance; TPH1, tryptophan hydroxylase 1; MAO, monoamine oxidase; COMT, catechol-O-methyltransferase; eQTL, expression quantitative trait locus; CNS, central nervous system.

\section{Acknowledgments}

We would like to thank the Ilustre Colegio Oficial de Farmacéuticos de Málaga (Official Pharmacists' Association, Malaga) for their invaluable help with the encapsulation process.

\section{Author Contributions}

All authors contributed to data analysis, drafting or revising the article, have agreed on the journal to which the article will be submitted, gave final approval of the version to be published, and agree to be accountable for all aspects of the work.

\section{Funding}

This project had no specific funding.

\section{Disclosure}

The authors report no conflicts of interest in this work.

\section{References}

1. Young VR, Hussein MA, Murray E, Scrimshaw NS. Plasma tryptophan response curve and its relation to tryptophan requirements in young adult men. $J$ Nutr. 1971;101:45-59. doi:10.1093/jn/101.1.45

2. Richard DM, Dawes MA, Mathias CW, Acheson A, Hill-Kapturczak N, Dougherty DM. 1-tryptophan: basic metabolic functions, behavioral research and therapeutic indications. Int $J$ Tryptophan Res. 2009;2:45-60. doi:10.4137/IJTR.S2129

3. McCorvy JD, Roth BL. Structure and function of serotonin G protein-coupled receptors. Pharmacol Ther. 2015;150:129-142. doi:10.1016/j.pharmthera.2015.01.009

4. Waraich P, Goldner EM, Somers JM, Hsu L. Prevalence and incidence studies of mood disorders: a systematic review of the literature. Can J Psychiatry. 2004;49:124-138. doi:10.1177/070674370404900208

5. Cleare A, Pariante CM, Young AH, et al. Evidence-based guidelines for treating depressive disorders with antidepressants: a revision of the 2008 British Association for Psychopharmacology guidelines. J Psychopharmacol. 2015;29:459-525. doi:10.1177/0269881115581093
6. Morrissette DA, Stahl SM. Modulating the serotonin system in the treatment of major depressive disorder. CNS Spectrums. 2014;19 (S1):57-68. doi:10.1017/S1092852914000613

7. Mulinari S. Monoamine theories of depression: historical impact on biomedical research. J Hist Neurosci. 2012;21:366-392. doi:10.1080/ 0964704X.2011.623917

8. Hindmarch I. Beyond the monoamine hypothesis: mechanisms, molecules and methods. Eur Psychiatry. 2002;17:294-299. doi:10.1016/ S0924-9338(02)00653-3

9. Melander H, Salmonson T, Abadie E, van Zwieten-boot B. A regulatory apologia-A review of placebo-controlled studies in regulatory submissions of new-generation antidepressants. Eur Neuropsychopharmacol. 2008;18:623-627. doi:10.1016/j. euroneuro.2008.06.003

10. Walsh BT, Seidman SN, Sysko R, Gould M. Placebo response in studies of major depression: variable, substantial, and growing. JAMA. 2002;287:1840-1847. doi:10.1001/jama.287.14.1840

11. Owens MJ. Selectivity of antidepressants: from the monoamine hypothesis of depression to the SSRI revolution and beyond. $J$ Clin Psychiatry. 2004;65:5-10.

12. Lauer JW. Biochemical and clinical changes resulting from administration of marsilid and tryptophan to schizophrenic patients; the clinical aspects. J Clin Exp Psychopathol. 1958;19:110-113.

13. Coppen A, Shaw DM, Farrell JP. Potentiation of the antidepressive effect of a monoamine-oxidase inhibitor by tryptophan. Lancet. 1963;7272:79-81. doi:10.1016/S0140-6736(63)91084-5

14. Glassman AH, Platman SR. Potentiation of a monoamine oxidase inhibitor by tryptophan. J Psychiatr Res. 1969;7:83-88. doi:10.1016/ 0022-3956(69)90013-2

15. Pare CM. Potentiation of monoamino oxidase inhibitors by tryptophan. Lancet. 1963;7(2):527-528. doi:10.1016/S0140-6736(63)90271-X

16. Chouinard G. Bupropion and amitriptyline in the treatment of depressed patients. J Clin Psychiatry. 1983;44:121-129.

17. Markus CR. Dietary amino acids and brain serotonin function; implications for stress-related affective changes. Neuromolecular Med. 2008;10:247-258. doi:10.1007/s12017-008-8039-9

18. Markus R, Panhuysen G, Tuiten A, Koppeschaar H. Effects of food on cortisol and mood in vulnerable subjects under controllable and uncontrollable stress. Physiol Behav. 2000;70:333-342. doi:10.1016/ S0031-9384(00)00265-1

19. Andrade-Fernandez EM, Arce-Fernandez C, Seaone-Pesqueira G. Adaptación al español del cuestionario «Perfil de los Estados de Ánimo» en una muestra de deportistas. Psicothema. 2000;214:708-713.

20. Goldberg LR. Differential attribution to trait-descriptive terms to oneself as compared to well-liked, neutral and disliked others: a psychometric analysis. J Pers Soc Psychol. 1978;26:1012-1028. doi:10.1037/0022-3514.36.9.1012

21. Fernstrom JD. A perspective on the safety of supplemental tryptophan based on its metabolic fates. J Nutr. 2016;146:2601S-2608S. doi: $10.3945 /$ jn. 115.228643

22. Jenkins TA, Nguyen JCD, Polglaze KE, et al. Influence of tryptophan and serotonin on mood and cognition with a possible role of the gut-brain axis. Nutrients. 2016;8:56. doi:10.3390/nu8010056

23. Young SN, Teff KL. Tryptophan availability, 5HT synthesis and 5HT function. Prog Neuropsychopharmacol Biol Psychiatry. 1989;13:373. doi:10.1016/0278-5846(89)90126-7

24. Garpenstrand H, Ekblom J, Forslund K, Rylander G, Oreland L. Platelet monoamine oxidase activity is related to MAOB intron 13 genotype. J Neural Transm. 2000;107:523-530. doi:10.1007/ s007020070075

25. Jansson M, McCarthy S, Sullivan PF, et al. MAOA haplotypes associated with thrombocyte-MAO activity. BMC Genet. 2005;6:46. doi:10.1186/1471-2156-6-46

26. Gibson EL. Tryptophan supplementation and serotonin function: genetic variations in behavioural effects. Proc Nutr Soc. 2018;77:174-188. doi:10.1017/S0029665117004451 


\section{Publish your work in this journal}

The International Journal of General Medicine is an international, peer-reviewed open-access journal that focuses on general and internal medicine, pathogenesis, epidemiology, diagnosis, monitoring and treatment protocols. The journal is characterized by the rapid reporting of reviews, original research and clinical studies across all disease areas. The manuscript management system is completely online and includes a very quick and fair peer-review system, which is all easy to use. Visit http://www.dovepress.com/ testimonials.php to read real quotes from published authors. 